DFTT 72/98

ITFA $98-42$

MS-TPI-98-22

September 1999

\title{
Universal amplitude ratios in the 2D four state Potts model
}

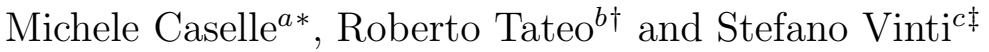 \\ a Dipartimento di Fisica Teorica dell'Università di Torino and \\ Istituto Nazionale di Fisica Nucleare, Sezione di Torino \\ via P.Giuria 1, I-10125 Torino, Italy \\ ${ }^{b}$ Universiteit van Amsterdam, Instituut voor Theoretische Fysica, \\ 1018 XE Amsterdam, The Netherlands \\ ${ }^{c}$ Institut für Theoretische Physik I, Universität Münster, \\ Wilhelm-Klemm-Str. 9, D-48149 Münster, Germany
}

\begin{abstract}
We present a Monte Carlo study of various universal amplitude ratios of the two dimensional $q=4$ Potts model. We simulated the model close to criticality in both phases taking care to keep the systematic errors, due to finite size effects and logarithmic corrections in the scaling functions, under control. Our results are compatible with those recently obtained using the form-factors approach and with the existing low temperature series for the model.
\end{abstract}

PACS: 75.10.H , 11.10.J

Keywords: 4-state Potts, Monte Carlo, universal amplitude ratios.

\footnotetext{
*e-mail: caselle@to.infn.it

†e-mail: tateo@wins.uva.nl

$\ddagger$ e-mail: vinti@uni-muenster.de
} 


\section{Introduction}

One of the peculiar features of the two dimensional four state Potts model is the presence of a marginal field which leads to universal multiplicative logarithmic corrections to the scaling laws.

These corrections can be exactly evaluated [1], 2] but, as often happens when dealing with marginal fields, they are accompanied by still large subleading non-universal contributions which completely mask the scaling behaviour of the system (at least for those values of the correlation length which can be reached in standard simulations).

This is different from the behaviour, for instance, of the 3d Ising model where even at moderate values of the correlation length, the non-universal corrections [3] give very small contributions; thus they can be safely taken into account by adding to the scaling functions only the first non-universal term.

In the present case, instead, these corrections are so large that the reliability of the fits, in which only the first subleading term is taken into account, becomes questionable. On the other hand, with current numerical precision, it is almost impossible to add further corrections without losing any predictive power in the fits.

This makes the numerical study of the four state Potts model one of the most difficult tasks in the context of Monte Carlo simulations of two dimensional spin models.

This problem was recently addressed by J.Salas and A.D.Sokal in [4 by extending the RG analysis of [1], 2] up to third order in the fields. They succeeded in obtaining the universal leading corrections to the scaling, which turn out to be additive terms of the generic form $\log \log / \mathrm{log}$. The improved scaling functions were then tested by looking at the critical finite size properties of the model, and an improvement of the scaling behaviour of the data was observed which however turned out to be still affected by large non-universal $1 / \log$ terms.

In this paper we return to this problem by looking at various universal amplitude combinations of the model [5]. We find an improvement in the scaling behaviour of our data if the universal contributions evaluated in [4] are taken into account.

However, as in [4], this is not enough to describe the data; non-universal corrections must be considered and final results crucially depend on the type 
of terms included in the scaling functions. We consider this one of the most delicate aspects of this paper. For this reason we described, as precisely as possible, the procedure used to construct the scaling functions (see sect. 7) and included in the paper, besides final estimates for the amplitude ratios, also the direct results of the Monte Carlo simulation (see tables 1,2 and 3) so that the reader can use the data to study alternative scaling functions and possibly find a clever way to control the systematic errors involved in the truncation that we suggest.

Fortunately for the present problem we have an independent way to test our results. In fact, thanks to a recent work by J.L.Cardy and G.Delfino [6], precise estimates for amplitude combinations are now available in the continuum limit. The relatively good agreement found between our estimates and those of [6] make us confident of the reliability of our results and at the same time strongly supports the correctness of the form-factors derivation of ref. 6.

This paper is organized as follows. In sect. 2 we give a general introduction to the $q$-state Potts model and we summarize a few known facts concerning the phase diagram and its scaling limit description in the framework of conformal and perturbed conformal field theory. In sects. 3 and 4 we introduce and discuss the observables and the amplitude ratios in which we are interested. In sect. 5 and 6 we describe the Monte Carlo simulation and test the results by comparing them with existing low temperature series and by imposing the duality relations on the internal energy and the specific heat.

Sect. 7 is devoted to the study of the scaling behaviour of the observables and to extract the best estimates for the amplitude combinations. Sect. 8 is devoted to a comparison of the results with those of ref. [6] while in sect. 9 we have collected some concluding remarks.

\section{The Model}

We study the four state Potts model in two dimensions on a simple square lattice. The action is given by

$$
S_{P o t t s}=-\beta \sum_{<x, y>} \delta_{s(x), s(y)}
$$

where the field variable $s(x)$ takes the values $0,1,2,3 ; \quad x \equiv\left(x_{0}, x_{1}\right)$ labels the sites of the lattice and the notation $\langle x, y\rangle$ indicates that the sum is taken 
on nearest neighbor sites only. The $\delta$ function is defined as usual: $\delta_{a, b}=1$ if $a=b$ and 0 otherwise. The coupling $\beta$ is related to the temperature in the standard way $\beta \equiv \frac{1}{k T}$. In the following we shall always consider lattices of equal extension $L$ and periodic boundary conditions in both directions.

Several results are known exactly for this model. The action eq. (11), is invariant under the permutation group $S_{4}$. However in the low temperature phase this symmetry is spontaneously broken to $S_{3}$. The two phases are related by duality and separated by a second order phase transition located at $\beta_{c} \equiv \frac{1}{k T_{c}}=\log (3)=1.098612$. The dual coupling $\tilde{\beta}$ is related to the original coupling $\beta$ by

$$
\tilde{\beta}=-\log \left(\frac{1-e^{-\beta}}{1+3 e^{-\beta}}\right),
$$

and the fixed point of this relation is the critical coupling $\beta_{c}$.

It is useful to introduce the variables

$$
\sigma_{\alpha}(x)=\delta_{s(x), \alpha}-\frac{1}{4} \quad, \quad \alpha=0,1,2,3 \quad .
$$

It is easy to see that $\left\langle\sigma_{\alpha}\right\rangle=0 \forall \alpha$ in the high temperature phase and that they all become different from zero in the low temperature phase. In particular for one of the four values of the spin (which we shall call in the following "majority spin") $\left\langle\sigma_{\alpha}\right\rangle>0$, while for the three other values we have $\left\langle\sigma_{\alpha}\right\rangle<0$.

It has been shown (cf. [8]) that the partition function $Z(T, q)$ for the $q$-state Potts models ( $q$ integer) on a square lattice $\Lambda$ can be written as

$$
Z(T, q)=\sum_{\{G\}}\left(e^{\beta}-1\right)^{N} q^{\nu}
$$

The sum in eq. (田) runs over all the graphs $G$ on $\Lambda, C$ is the number of connected components (including isolated sites) in $G$, and $N$ is the number of bounds on the lattice edges. For a better understanding of some of the peculiar features of the four-state Potts model it is convenient to consider the phase diagram of the whole family of models, defined for arbitrary $q>$ 0 . Eq. (4) provides an expression suitable for extending the definition of $Z(T, q)$ to non integer values of $q$. The $q$-state Potts model undergoes a phase transition at

$$
\beta_{c}=\log (\sqrt{q}+1)
$$


Below this temperature the system is in its $S_{q}$-broken symmetry phase whereas above it the system is fully disordered. The transition at $T=T_{c}$ is first order for $q>4$ but becomes second order for $q \leq 4$, in the latter case the model gets renormalized into a conformal field theory with central charge [9]

$$
c=1-\frac{6}{(l-1) l},
$$

where $l$ is related to $q$ by

$$
\frac{2 \pi}{l}=\arctan \left(\frac{\sqrt{4 q-q^{2}}}{(q-2)}\right)
$$

In the scaling limit, at rational points $l=p /\left(p^{\prime}-p\right)$, the thermal field $\varepsilon$ rescales with scaling dimension 10

$$
\Delta_{\varepsilon}=\frac{1}{2}\left(1+\frac{3}{l-1}\right),
$$

hence it can be identified with the operator $\phi_{21}$ in the $M_{p, p^{\prime}}$ minimal conformal model. Notice also that eq. (7) shows that two square-root branch points at $q=0$ and $q=4$ are present. At $q=0$ the thermal operator is marginal, in the analytically-continued second branch it becomes irrelevant and the critical point has moved into the antiferromagnetic region. The physics in this sector is certainly very interesting but it is slightly less relevant for our current interests. More related to the subject of this paper is, instead, the physical meaning of the second branch point at $q=4$. Let us consider a further variant of the model in which vacancies are allowed, and correspondingly a chemical potential $\mu$ is introduced. In the sector $0<q<4$ with $\mu$ negative or sufficiently small, the additional dilution field turns out to be irrelevant and the system still undergoes a second order phase transition in the same universal class of the pure Potts model. Near the transition point the dilution field scales with a conformal dimension

$$
\Delta_{\mu}=2+\frac{4}{l-1}
$$

and it can be identified with primary conformal operator $\phi_{31}$.

At $q=4$, the dilution field $\phi_{\mu}$ becomes marginal; along the critical RG flow its slow rate of disappearance cause now multiplicative logarithm corrections to the critical behaviour. From eq. (7) we also see that the net result 
of the entrance in the second branch consists of a negation of $l$. Hence at the same value of $q$, but on the second branch we now have a conformal field theory (CFT) with central charge

$$
c=1-\frac{6}{l(l+1)},
$$

with thermal and dilution fields with dimensions

$$
\Delta_{\varepsilon}=\frac{1}{2}\left(1-\frac{3}{l+1}\right) \quad, \quad \Delta_{\mu}=2-\frac{4}{l+1} .
$$

These two fields can now be respectively identified with the relevant conformal operators $\phi_{12}$ and $\phi_{13}$. In conclusion, at fixed $q<4$, the phase diagram in the plane $(\mu, T)$ is as follows: if $\mu$ is negative or sufficiently small then at $T=T_{c}(\mu)$ the system undergoes a second order phase transition in the universal class of the pure Potts model, whereas when $\mu$ is large the transition becomes of the first order. On the critical line $\left(\mu, T_{c}(\mu)\right)$ the point marking the change of critical behavior is the tricritical point. From the scaling quantum field theory point of view, the picture looks also consistent with the one described above. First notice that perturbation of the conformal field theory with either the thermal or the dilution field is integrable [11] and that the associated quantum field theories have been the subject of very deep studies (see for example [12, 13, 14, 15, 16, 17]). The thermal operator $\varepsilon$ drives the system into a massive ordered or disordered phase depending on the sign of the perturbing parameter [15, 16, 17]. The operator $\phi_{\mu}$ instead moves the tricritical model either into a massive phase (a line of first-order transitions) or into a critical massless phase. The IR fixed point of the latter is the Potts-model CFT and the two less irrelevant attracting operators are the fields $\phi_{31}$ and $T \bar{T}$ [15, 16, 17].

Quantum reductions of the Izergin-Korepin model at rational points, giving the S-matrix elements for the $\phi_{12} / \phi_{21}$ perturbations, were first obtained by F.A.Smirnov [12]. Subsequently using a somehow different kink basis, L.Chim and A.B.Zamolodchikov [18] defined alternative scattering elements for the model. The latter formulation, being more suitable for analytic continuation at arbitrary values of $q$, has been used by J.L.Cardy and G.Delfino [6] to make predictions about the values of some universal amplitudes. The method used is a variant of the form-factors approach to the correlation functions proposed in 119 . 


\section{The observables}

\subsection{Magnetization}

The magnetization of a given configuration is defined as:

$$
m=\frac{1}{V} \sum_{x} \sigma_{\alpha_{m}}(x)
$$

where $V \equiv L^{2}$ is the volume of the lattice and $\alpha_{m}$ is the value of spin corresponding to the majority of the spins. However, in a finite volume at arbitrary finite low temperature the $S_{4}$ symmetry of the model is not spontaneously broken. Practically this means that, in the simulation sample, configurations with all the four possible values of $\alpha_{m}$ appear with equal probability. In order to obtain a low temperature non-vanishing magnetization a magnetic field $h$ that explicitly breaks the symmetry, must be coupled to the system. The thermodynamic limit at non zero $h$ should be taken first, then the limit of vanishing magnetic field could be performed. However it is difficult to follow this route in a numerical study. An alternative, commonly adopted, approach is to identify $\alpha_{m}$ in each configuration by counting the spins belonging to the four possible values of $\alpha$ and then extracting the majority one. This procedure works in a satisfactory way if the lattice size and coupling constants are such that the probability of finding interfaces among different vacua is negligible. We carefully chose our lattice sizes so as to satisfy this bound 母.

In the following we shall assume for simplicity that $\alpha_{m}=0$ is the value of the majority spin and shall denote the remaining three values with roman indices $i, j, k, \ldots=1,2,3$.

Close to criticality and at $t \equiv \frac{\beta_{c}-\beta}{\beta_{c}}<0$, the magnetization scales as [4]

\footnotetext{
${ }^{1}$ Let us note, as a side remark, that this procedure, in the Ising case is equivalent to the choice

$$
<m>\equiv \lim _{L \rightarrow \infty}<|m|>\quad\left(m=\frac{1}{V} \sum_{i} s_{i}\right) \quad,
$$

where the $s_{i}$ 's are in this case Ising spins.

The finite size behaviour of this observable was carefully studied in 20]. It was shown that this choice converges to the infinite volume value better than any other existing proposal and that the asymptotic, infinite volume, value is reached for lattices of size $L>\sim 8 \xi$, where $\xi$ denotes the correlation length. In our simulations we always used lattice sizes much larger than this threshold.
} 


$$
<m>\sim B(-t)^{\frac{1}{12}}(-\log (-t))^{-\frac{1}{8}}\left[1-\frac{3}{16} \frac{\log (-\log (-t))}{-\log (-t)}+O\left(\frac{1}{\log (-t)}\right)\right]
$$

\subsection{Magnetic susceptibility}

The susceptibility

$$
\chi=\frac{\partial<m>}{\partial H}
$$

gives the response of the magnetization to an external magnetic field and it can be expressed in terms of moments of the magnetization

$$
\chi=V\left(<m^{2}>-<m>^{2}\right) .
$$

In the high temperature phase this means

$$
\chi=V<m^{2}>\quad=V<\sigma_{\alpha}^{2}>,
$$

where $\alpha$ is any one of the four values $(0,1,2,3))^{2}$.

In the broken symmetric phase, depending on the choice of coupling the external magnetic field to the majority spin or to one (or more) of the other values, two kinds of susceptibilities can be defined [22]

$$
\begin{array}{ll}
\chi_{l}=\left\langle\sigma_{0}^{2}\right\rangle & \text { longitudinal } \\
\chi_{t}=\left\langle\left(\sigma_{i}-\sigma_{j}\right)^{2}\right\rangle \quad(i \neq j) & \text { transverse } .
\end{array}
$$

In this paper we concentrate on the longitudinal susceptibility $\chi_{l}$.

\footnotetext{
${ }^{2}$ Notice however that, inspired by the analogy with the Ising model or by the embedding in the AT model, different definitions of the order parameter (hence of the mean magnetization in the symmetric phase) are possible. For instance

$$
<m_{1}>=<\sigma_{\alpha}-\sigma_{\beta}>
$$

with $\alpha \neq \beta$ or

$$
<m_{2}>=<\sigma_{\alpha}-\sigma_{\beta}+\sigma_{\gamma}-\sigma_{\delta}>
$$

with $\alpha \neq \beta \neq \gamma \neq \delta$. The corresponding susceptibilities are related to $\chi$ by simple multiplicative constants. In comparing our results with those of [21, 4] one must take into account this different normalization.
} 
Close to the critical temperature $\chi$ and $\chi_{l}$ scale as

$$
\begin{gathered}
\chi \sim \Gamma_{+}(t)^{-\frac{7}{6}}(-\log |t|)^{\frac{3}{4}}\left[1+\frac{9}{8} \frac{\log (-\log |t|)}{-\log |t|}+O\left(\frac{1}{\log |t|}\right)\right] \quad(t>0) \\
\chi_{l} \sim \Gamma_{-}(-t)^{-\frac{7}{6}}(-\log |t|)^{\frac{3}{4}}\left[1+\frac{9}{8} \frac{\log (-\log |t|)}{-\log |t|}+O\left(\frac{1}{\log |t|}\right)\right] \quad(t<0) .
\end{gathered}
$$

\subsection{Internal energy and specific heat}

The internal energy is defined as

$$
E=\frac{1}{2 V} \sum_{<x, y>} \delta_{s(x), s(y)},
$$

the specific heat

$$
C \equiv \frac{d<E>}{d \beta}=2 V\left(<E^{2}>-<E>^{2}\right)
$$

Duality relates both internal energy and specific heat in the low temperature phase to those in the high temperature phase. The relations are

$$
\begin{gathered}
\left(1-e^{-\beta}\right) E(\beta)=1-\left(1-e^{-\tilde{\beta}}\right) E(\tilde{\beta}) \\
C(\beta)\left(1-e^{-\beta}\right)^{2}+e^{-\beta}\left(1-e^{-\beta}\right) E(\beta)=C(\tilde{\beta})\left(1-e^{-\tilde{\beta}}\right)^{2}+e^{-\tilde{\beta}}\left(1-e^{-\tilde{\beta}}\right) E(\tilde{\beta}) .
\end{gathered}
$$

Close to the critical temperature we have

$$
\begin{gathered}
C \sim A_{+}(t)^{-\frac{2}{3}}(-\log |t|)^{-1}\left[1-\frac{3}{2} \frac{\log (-\log |t|)}{-\log |t|}+O\left(\frac{1}{\log |t|}\right)\right] \quad(t>0), \\
C \sim A_{-}(-t)^{-\frac{2}{3}}(-\log |t|)^{-1}\left[1-\frac{3}{2} \frac{\log (-\log |t|)}{-\log |t|}+O\left(\frac{1}{\log |t|}\right)\right] \quad(t<0) .
\end{gathered}
$$

\footnotetext{
${ }^{3}$ Notice that there is a misprint in the analogue of this equation in ref. 㘬.
} 


\subsection{Second moment correlation length}

We consider the decay of so-called time-slice correlation functions. The magnetization of a time-slice is given by

$$
S_{\alpha}\left(x_{0}\right)=\frac{1}{L} \sum_{x_{1}} \sigma_{\alpha}\left(x_{0}, x_{1}\right)
$$

Let us define the correlation function

$$
G_{\alpha \beta}(\tau)=\sum_{x_{0}}\left\{\left\langle S_{\alpha}\left(x_{0}\right) S_{\beta}\left(x_{0}+\tau\right)\right\rangle-\left\langle S_{\alpha}\left(x_{0}\right)\right\rangle\left\langle S_{\beta}\left(x_{0}\right)\right\rangle\right\} \quad .
$$

For any choice of the indices $\alpha$ and $\beta$ in (26), the dominant large distance behaviour of $G(\tau)$ is dominated by the lowest mass of the model:

$$
G(\tau) \propto \exp (-\tau / \xi),
$$

where $\xi$ is the exponential correlation length and coincides with the inverse of the lowest mass of the model. However, at low temperature, the rich structure of the spectrum (cf. [6]) can mask this asymptotic behaviour. For this reason, in the following, we shall concentrate on $G_{00}$ which has the "neatest" asymptotic behaviour. This will also allow us to directly compare our results with those of [6]. In the high temperature phase the lowest mass is well separated from all other excitations in the spectrum and extracting the exponential correlation length is much simpler.

Close to criticality the behaviour of the correlation length is governed by the scaling laws

$$
\begin{gathered}
\xi \sim f_{+}(t)^{-\frac{2}{3}}(-\log |t|)^{\frac{1}{2}}\left[1+\frac{3}{4} \frac{\log (-\log |t|)}{-\log |t|}+O\left(\frac{1}{\log |t|}\right)\right] \quad(t>0) \\
\xi \sim f_{-}(-t)^{-\frac{2}{3}}(-\log |t|)^{\frac{1}{2}}\left[1+\frac{3}{4} \frac{\log (-\log |t|)}{-\log |t|}+O\left(\frac{1}{\log |t|}\right)\right] \quad(t<0) .
\end{gathered}
$$

We are also interested in the second moment correlation length $\xi_{2}$, to evaluate $\xi_{2}$ we used the estimator [21, [4]

$$
\xi^{(2)} \equiv \frac{\left(\frac{\chi}{F}-1\right)^{\frac{1}{2}}}{2 \sin (\pi / L)},
$$


where $\chi$ is the susceptibility and $F$ is the Fourier transform of the correlation function at the smallest nonzero momentum $(2 \pi / L, 0)$.

Notice that the susceptibility can be rewritten as zero momentum Fourier transform of the correlation function, hence, in order to have a consistent definition, the same correlation function must be chosen in both $\chi$ and $F$. In the low temperature regime we are interested in setting the longitudinal susceptibility

$$
\chi_{l} \equiv \sum_{x} G_{0,0}(x)
$$

in eq. (29), hence, we shall study

$$
F \equiv \sum_{x} e^{2 i \pi x_{1} / L} G_{0,0}(x)
$$

$\xi_{2}$ is a very popular approximation for the exponential correlation length since, in Monte Carlo simulations, its numerical evaluation is much simpler than that of $\xi$.

Moreover it is the length scale which is directly observed in scattering experiments. It is important to stress that $\xi_{2}$ and $\xi$ are not fully equivalent (cf. [3] ), even though their critical behaviours are the same up to a multiplicative factor. In particular, the ratio $\xi / \xi_{2}$ gives an idea of the density of the lowest states of the spectrum. If the lower excited states are well separated the ratio is almost one, whereas a significantly bigger ratio indicate a denser distribution of states. Furthermore, different choices of the correlation function in eq.s (30,31) lead to different values of $\xi_{2}$, while the exponential correlation length is always the same. A careful study of these differences can give several information on the spectrum of the theory. We shall call the critical amplitudes $f_{2, \pm}$ for $\xi_{2}$ to distinguish them from $f_{ \pm}$.

\section{Amplitude ratios}

We are interested in the following amplitude ratios

$$
R_{\chi}=\frac{\Gamma_{+}}{\Gamma_{-}} \quad, \quad R_{\xi, 2}=\frac{f_{2,+}}{f_{2,-}}
$$

and the following amplitude combinations

$$
R_{1}=\frac{\Gamma_{-}}{f_{2,-}^{2} B^{2}} \quad, \quad R_{2}=\frac{\Gamma_{+}}{f_{2,+}^{2} B^{2}},
$$


which are scale invariant thanks to the (hyper)scaling relations among the critical exponents

$$
\alpha+2 \beta+\gamma=2 \quad, \quad d \nu=2-\alpha \quad .
$$

We are also interested in the combinations

$$
\begin{array}{lll}
R_{c}^{+}=\frac{A_{+} \Gamma_{+}}{B^{2}} \quad, & R_{\xi}^{+}=\sqrt{A_{+}} f_{2,+}, \\
R_{c}^{-}=\frac{A_{-} \Gamma_{-}}{B^{2}} \quad, & R_{\xi}^{-}=\sqrt{A_{-}} f_{2,-},
\end{array}
$$

which have particularly interesting behaviours in the 4 state Potts model (see below). We shall neglect the amplitude ratio $A_{+} / A_{-}$which is trivially 1 due to duality f.

\section{The simulations}

We produced a standard cluster algorithm using both the Wolff single cluster update and the Swendson Wang cluster update. After preliminary tests, we used the latter algorithm for our high statistic simulations.

To check our program we made comparisons of the MC results with the exact solution on a $3^{2}$ lattice and with the Salas and Sokal (ref.s [21, , 4) results at the critical point on a $16^{2}$ lattice, with a comparable statistics.

We simulated the 4 state Potts model in the high and low temperature phases for 16 values of the couplings which were chosen exactly as dual pairs. This choice allowed us first, to perform a very stringent test on our estimates for the thermal observables which must be related by duality and second, to obtain a direct estimate of some amplitude ratios. The results for the observables in which we are interested are reported in tables 1,2 and 3 .

Lattice sizes were chosen large enough to make finite size effects negligible within our statistical errors. After a preliminary test on the finite size behaviour of our observables we chose $L>10 \xi$ in the high temperature phase and $L>20 \xi$ in the low temperature phase, a default size $L=120$ was taken for small values of $\xi$. In each simulation the number of measurements was $2 \cdot 10^{7}$. Each measurement was separated from the next one by

\footnotetext{
${ }^{4}$ In principle this result could be used to test our simulation. But such test is completely equivalent to the test of the duality relation eq. (23) that we perform in tables 4 and 5 .
} 
two Swendsen-Wang updates. A standard jacknife procedure has been used to analyze statistical errors.

\begin{tabular}{|c|c|c|c|c|c|}
\hline$\beta$ & $L$ & $\xi_{2 n d}$ & $E$ & $C$ & $\chi$ \\
\hline 1.06722 & 120 & $6.44(5)$ & $0.64604(6)$ & $1.571(12)$ & $11.83(2)$ \\
1.07722 & 120 & $8.46(4)$ & $0.66318(6)$ & $1.892(13)$ & $18.78(3)$ \\
1.07972 & 120 & $9.25(3)$ & $0.66808(6)$ & $2.018(14)$ & $21.80(4)$ \\
1.08222 & 120 & $10.26(3)$ & $0.67337(6)$ & $2.189(15)$ & $25.90(5)$ \\
1.08472 & 120 & $11.60(3)$ & $0.67909(6)$ & $2.405(15)$ & $31.69(6)$ \\
1.08722 & 180 & $13.37(5)$ & $0.68544(6)$ & $2.614(22)$ & $40.31(9)$ \\
1.08972 & 180 & $15.94(5)$ & $0.69232(6)$ & $3.033(24)$ & $54.05(13)$ \\
1.09222 & 240 & $20.33(7)$ & $0.70056(6)$ & $3.574(35)$ & $80.88(24)$ \\
\hline
\end{tabular}

Table 1: Results in the high temperature phase.

\begin{tabular}{|c|c|c|c|c|}
\hline$\beta$ & $L$ & $\xi_{2 n d}$ & $E$ & $C$ \\
\hline 1.130500 & 120 & $2.85(5)$ & $0.850888(5)$ & $1.3718(10)$ \\
1.120231 & 120 & $3.69(5)$ & $0.835061(7)$ & $1.7474(16)$ \\
1.117684 & 120 & $4.00(4)$ & $0.830453(8)$ & $1.8851(17)$ \\
1.115135 & 120 & $4.45(4)$ & $0.825436(8)$ & $2.0516(20)$ \\
1.112597 & 120 & $5.01(4)$ & $0.820001(9)$ & $2.2604(24)$ \\
1.110065 & 120 & $5.77(4)$ & $0.813951(10)$ & $2.5274(28)$ \\
1.107540 & 120 & $6.98(4)$ & $0.807100(12)$ & $2.9048(40)$ \\
1.105020 & 180 & $8.89(7)$ & $0.799095(9)$ & $3.4872(49)$ \\
\hline
\end{tabular}

Table 2: Results in the low temperature phase: thermal observables and correlation lengths.

\section{Analysis of the results}




\begin{tabular}{|c|c|c|c|}
\hline$\beta$ & $L$ & $m$ & $\chi_{l}$ \\
\hline 1.130500 & 120 & $0.633288(7)$ & $1.2180(9)$ \\
1.120231 & 120 & $0.612874(11)$ & $2.1072(23)$ \\
1.117684 & 120 & $0.606331(13)$ & $2.5080(26)$ \\
1.115135 & 120 & $0.598834(14)$ & $3.0572(36)$ \\
1.112597 & 120 & $0.590219(16)$ & $3.8447(51)$ \\
1.110065 & 120 & $0.579950(19)$ & $5.0315(72)$ \\
1.107540 & 120 & $0.567272(26)$ & $7.053(14)$ \\
1.105020 & 180 & $0.550686(23)$ & $10.987(19)$ \\
\hline
\end{tabular}

Table 3: Results in the low temperature phase: magnetic observables.

\subsection{Energy and specific heat}

By using eq. (22) we have a non trivial test of our estimates both for the energy and for the specific heat. In table 4 we compare the results for the internal energy in the high temperature phase with those obtained using eq. (22) and the values measured with the dual coupling, at low temperature as input. A similar comparison for the specific heat can be found in table 5 .

\subsection{Magnetization and Susceptibility}

In tables 6 and 7 we compare our results for the magnetization and the low temperature longitudinal susceptibility with the series of ref. [23]. Both for the magnetization and for the susceptibility we used the diagonal Pade' approximant. As expected, the agreement, which is rather good far from the critical point becomes worse and worse as $\beta_{c}$ is approached. Notice however that we used the simplest possible resummation technique, more sophisticated approaches like the double biased IDA of ref. [24] could give better results and could also give a way to estimate the systematic errors involved in the truncation and resummation of the series (for an attempt in this direction in the case of the 3d Ising model see for instance [3]) In

\footnotetext{
${ }^{5}$ In comparing our values of the magnetization with those of ref. [23] one must notice that there is a factor $4 / 3$ between the two definition of magnetization. On the contrary
} 


\begin{tabular}{|c|c|c|}
\hline$\beta$ & HT & LT+eq.(22) \\
\hline 1.06722 & $0.64604(6)$ & $0.646061(5)$ \\
1.07722 & $0.66318(6)$ & $0.663179(7)$ \\
1.07972 & $0.66808(6)$ & $0.668074(8)$ \\
1.08222 & $0.67337(6)$ & $0.673365(8)$ \\
1.08472 & $0.67909(6)$ & $0.679055(9)$ \\
1.08722 & $0.68544(6)$ & $0.685341(10)$ \\
1.08972 & $0.69232(6)$ & $0.69240(1)$ \\
1.09222 & $0.70056(6)$ & $0.70060(1)$ \\
\hline
\end{tabular}

Table 4: Comparison between the internal energy measured in the simulation at high temperature (second column) and the values obtained, from the internal energy measured at low temperature, using the duality relation (22) (third column).

the case of the susceptibility the discrepancy between the results from the series expansion and our Monte Carlo are larger and only the first value of beta agree within the errors. It is clear however that we are pushing the series to their limit of validity and in fact, looking at nondiagonal Pade' approximants, one sees very large fluctuations (much larger than in the case of magnetization) in the series estimates.

\section{Scaling behaviour}

Let us now address the problem of extracting the continuum limit values of the quantities discussed in the previous section. As mentioned in the introduction, due to the presence of large corrections to scaling terms, this requires a rather non-trivial analysis. We followed a three step procedure.

1] As first test we tried to fit the data using only the dominant multiplicative $\log$ correction keeping into account for $C$ and $\chi$ the possible existence of bulk constant terms. Hence a one parameter fit for $\xi_{2}$ and $m$ and a two parameter fit for $C$ and $\chi$. In all cases we found very

there is complete agreement between the two definitions for the longitudinal susceptibility. 


\begin{tabular}{|c|c|c|}
\hline$\beta$ & HT & LT+eq.(匂) \\
\hline 1.06722 & $1.571(12)$ & $1.555(1)$ \\
1.07722 & $1.892(13)$ & $1.903(2)$ \\
1.07972 & $2.018(14)$ & $2.032(2)$ \\
1.08222 & $2.189(15)$ & $2.191(2)$ \\
1.08472 & $2.405(15)$ & $2.391(3)$ \\
1.08722 & $2.614(22)$ & $2.646(3)$ \\
1.08972 & $3.033(24)$ & $3.011(4)$ \\
1.09222 & $3.574(35)$ & $3.579(5)$ \\
\hline
\end{tabular}

Table 5: Comparison between the specific heat measured in the simulation at high temperature (second column) and the values obtained, from the internal energy and the specific heat measured at low temperature, using the duality relations 22,23) (third column).

high $\chi_{r}^{2}$ and, even eliminating all the data except the two couplings nearest to $\beta_{c}$, it was impossible to reach a reasonable confidence level. This clearly indicates that additive corrections to the scaling cannot be neglected.

2] The second step was to add the first non-universal correction, (that of the form $1 / \log )$. At the same time we also included the universal corrections evaluated by Salas and Sokal, which are of the same order of magnitude and do not add degrees of freedom in the fit. The resulting fitting functions are (see sect. 3):

$$
\begin{aligned}
& \chi(t)=a_{0}+\Gamma_{ \pm}|t|^{-\frac{7}{6}}(-\log |t|)^{\frac{3}{4}}\left[1+\frac{9}{8} \frac{\log (-\log |t|)}{-\log |t|}+\frac{a_{1}}{-\log |t|}\right], \\
& \xi_{2}(t)=f_{2, \pm}|t|^{-\frac{2}{3}}(-\log |t|)^{\frac{1}{2}}\left[1+\frac{3}{4} \frac{\log (-\log |t|)}{-\log |t|}+\frac{a_{1}}{-\log |t|}\right], \\
& m(t)=B|t|^{\frac{1}{12}}(-\log |t|)^{-\frac{1}{8}}\left[1-\frac{3}{16} \frac{\log (-\log |t|)}{-\log |t|}+\frac{a_{1}}{-\log |t|}\right] .
\end{aligned}
$$




\begin{tabular}{|c|c|c|}
\hline$\beta$ & our MC & series \\
\hline 1.130500 & $0.633288(7)$ & 0.633275 \\
1.120231 & $0.612874(11)$ & 0.612863 \\
1.117684 & $0.606331(13)$ & 0.606310 \\
1.115135 & $0.598834(14)$ & 0.598853 \\
1.112597 & $0.590219(16)$ & 0.590268 \\
1.110065 & $0.579950(19)$ & 0.580154 \\
1.107540 & $0.567272(26)$ & 0.567899 \\
1.105020 & $0.550686(23)$ & 0.552456 \\
\hline
\end{tabular}

Table 6: Comparison of our Monte Carlo results for the magnetization with a Pade' resummation of the series of ref. [23] .

$$
C(t)=a_{0}+A_{ \pm}|t|^{-\frac{2}{3}}(-\log |t|)^{-1}\left[1-\frac{3}{2} \frac{\log (-\log |t|)}{-\log |t|}+\frac{a_{1}}{-\log |t|}\right],
$$

We performed these two (or three) parameter linear fits, first taking into account all the data and then systematically eliminating those farthest from the critical point until an acceptable reduced $\chi^{2}$ (namely a $\chi^{2}$ lower than 1) was reached. In all cases, except for the magnetization, with this second step we reached an acceptable C.L. and stopped. Notice that in most of the cases such acceptable C.L. could be reached keeping all the data. (see the second column of table 8). We also realized at this stage that the critical amplitudes $A_{ \pm}$could be obtained in a much more efficient way by looking at the internal energy data. We shall discuss this point in detail in the next subsection.

3] In the next step we added a next-to-leading non-universal correction. Among the various possible terms we chose the one giving, in the range of values of $\beta$ of our simulations, the largest contribution. We had to resort to this last step only for the magnetization. In this case there are two competing corrections. The first one is the $1 / \log ^{2}$ term: certainly expected due to the presence of the marginal field. But there is also second possibility: thanks to the CFT solution of the model we know 


\begin{tabular}{|c|c|c|}
\hline$\beta$ & our MC & series \\
\hline 1.130500 & $1.2180(9)$ & 1.2187 \\
1.120231 & $2.1072(23)$ & 2.1241 \\
1.117684 & $2.5080(26)$ & 2.5395 \\
1.115135 & $3.0572(36)$ & 3.1179 \\
1.112597 & $3.8447(51)$ & 3.9661 \\
1.110065 & $5.0315(72)$ & 5.3242 \\
1.107540 & $7.053(14)$ & 7.795 \\
1.105020 & $10.987(19)$ & 13.647 \\
\hline
\end{tabular}

Table 7: Comparison of our Monte Carlo results for $\chi_{l}$ with a Pade' resummation of the series of ref. 23] .

that in the spectrum there is a subleading magnetic operator to which corresponds a new critical index $\beta^{\prime}=3 / 4$.

The two terms have comparable magnitude, but it turns out that the subleading magnetic correction gives a slightly larger contribution in the range of interest. So, according to our strategy, we kept only this contribution and neglected the $1 / \log ^{2}$ one. The resulting scaling function is

$$
m(t)=B|t|^{\frac{1}{12}}(-\log |t|)^{-\frac{1}{8}}\left[1-\frac{3}{16} \frac{\log (-\log |t|)}{-\log |t|}+\frac{a_{1}}{-\log |t|}+a_{2}|t|^{\frac{2}{3}}\right] .
$$

Adding also the subleading magnetic term in the fit we found an impressive lowering of the $\chi^{2}$.

The results of these fits are summarized in table 8. It is important to stress that all the quoted errors are statistical. Besides them we also expect systematic errors due to the truncation of the scaling functions to $O(\log |t|)$ or, in the case of the magnetization, to the choice of the next-to-leading non-universal correction. In sect. 7.2 we shall discuss this problem in more detail. 


\begin{tabular}{|c|c|c|c|c|c|c|c|}
\hline Obs. & $N$ & $\chi_{r}^{2}$ & C.L. & Amplitude & $a_{0}$ & $a_{1}$ & $a_{2}$ \\
\hline$\chi_{+}$ & 8 & 0.93 & $46 \%$ & $\Gamma_{+}=0.0223(14)$ & $0.05(14)$ & $6.5(4)$ & \\
$\chi_{-}$ & 8 & 0.44 & $81 \%$ & $\Gamma_{-}=0.00711(10)$ & $0.02(1)$ & $-1.24(10)$ & \\
$\xi_{2,+}$ & 8 & 0.48 & $82 \%$ & $f_{2,+}=0.192(4)$ & & $1.35(11)$ & \\
$\xi_{2,-}$ & 7 & 0.44 & $82 \%$ & $f_{2,-}=0.088(4)$ & & $1.06(27)$ & \\
$\mathrm{m}$ & 8 & 0.80 & $54 \%$ & $B=1.1621(11)$ & & $-0.220(6)$ & $-0.144(9)$ \\
\hline
\end{tabular}

Table 8: Results of the fits for susceptibility, magnetization and correlation length. In the second column we report the number of data taken into account in the fit, in the third column the reduced chi square and in the fourth column the confidence level of the fit. The last four columns contain the best fit estimates of the parameters of the fit. For the magnetization we also have the contribution of a next to leading magnetization operator (see text) $a_{2}$.

\subsection{The critical amplitudes $A_{ \pm}$}

The most efficient way to obtain the critical amplitudes $A_{ \pm}$is to fit the internal energy (for which we have very precise data) instead of the specific heat. The bulk value of the energy is known (from duality) to be $E\left(\beta_{c}\right)=\frac{3}{4}$ however, due to the finite size of the lattice that we simulated we must account for possible small deviations from this asymptotic result. We end up with the following fitting function

$E(t)=\frac{3}{4}+a_{-1}+a_{0}|t|+3 A_{ \pm}|t|^{\frac{1}{3}}(-\log |t|)^{-1}\left[1-\frac{3}{2} \frac{\log (-\log |t|)}{-\log |t|}+\frac{a_{1}}{-\log |t|}\right]$

A severe constraint on the results of this fit is represented by duality which implies $A_{+}=A_{-}$. The result of the fits in the low and high $\mathrm{T}$ phases are reported in table 9 , where it can be seen that the values of $a_{0}$, and $A_{ \pm}$ extracted in the two phases are indeed compatible within the errors and that $a_{-1}$ is, as expected, very small. Combining the two estimates of $A_{+}$and $A_{-}$ we extract as our final estimate: $A_{ \pm}=1.30(6)$. 


\begin{tabular}{|c|c|c|c|c|c|c|c|}
\hline Obs. & $N$ & $\chi_{r}^{2}$ & C.L. & Amplitude & $a_{-1}$ & $a_{0}$ & $a_{1}$ \\
\hline$E_{+}$ & 7 & 0.67 & $57 \%$ & $A_{+}=1.29(5)$ & $-0.0001(29)$ & $3.0(1.3)$ & $-1.47(48)$ \\
$E_{-}$ & 7 & 0.87 & $45 \%$ & $A_{-}=1.316(9)$ & $-0.0056(5)$ & $1.30(18)$ & $-0.93(6)$ \\
\hline
\end{tabular}

Table 9: Results of the fits for the internal energy.

\subsection{Non-universal corrections}

A crucial ingredient to test the reliability of the above fits is given by the magnitude of the non-universal corrections. In the range of $\beta$ values that we studied the log of the reduced temperature $t$ takes values which range from -4 up to -5 . The non-linear contributions listed in tables 8 and 9 must be compared with this reference scale. One easily realizes that for all quantities these corrections are rather large (they are more or less of the same order of magnitude of the universal corrections evaluated in [四) and in the particular case of the high temperature susceptibility they are very large. This suggests that, even if the fits have a very good confidence level, caution is needed in assuming the best fit estimates for the amplitudes which could be affected by systematic deviations. Notice that there is no hope to control such large non-universal contributions by tuning $\beta$ towards the critical temperature. In fact it would be necessary to gain at least a factor 10 in $\log (\mathrm{t})$ which, as it can be easily seen, would imply a huge enhancement of $\xi$.

We tried to estimate the systematic errors which affect our estimates of the critical amplitude with the following method. We repeated the analysis discussed above adding in the fitting functions a term of the form $1 /(\log (t))^{2}$ (which in the range of values of $t$ that we study is the largest among the correction to scaling terms that we neglect) with amplitude equal in modulus to the $a_{1}$ amplitudes listed in tables 8 and 9 and with plus and minus sign. The two resulting values for the critical amplitude give a (admittedly rough) idea of the systematic deviations that we may expect in our estimates. The results are collected in table 10. By comparing with the statistical errors listed in tables 8 and 9 . one can see that the in all cases the systematic deviations are larger than the statistical errors. 


\begin{tabular}{|c|}
\hline Amplitude \\
\hline$\Gamma_{+}=0.0223(40)$ \\
$\Gamma_{-}=0.00711(30)$ \\
$f_{2,+}=0.192(10)$ \\
$f_{2,-}=0.088(6)$ \\
$B=1.1621(25)$ \\
$A_{ \pm}=1.30(10)$ \\
\hline
\end{tabular}

Table 10: Critical amplitudes with a tentative estimate of the systematic errors.

\subsection{Universal Amplitude ratios}

Plugging the values of the critical amplitudes quoted in table 10 in the definitions (32, 33, 35, 36) we find the values for the amplitude ratios reported in the second column of table 11. The errors quoted in table 11 have been obtained by using the systematic errors quoted in table 10 and discussed in sect 7.2 .

\section{Comparison with field theory predictions}

It is very interesting to compare our results with the $R_{\chi}, R_{\xi}, R_{c}^{+}$, and $R_{\xi}^{+}$ estimates obtained, using the S-matrix form-factors approach to the correlation functions, in [6]. It easy to obtain the remaining four ratios by using the following relations:

$$
R_{c}^{-}=\frac{R_{c}^{+}}{R_{\chi}} \quad, \quad R_{\xi}^{-}=\frac{R_{\xi}^{+}}{R_{\xi}} \quad, \quad R_{1}=\frac{R_{c}^{-}}{\left(R_{\xi}^{-}\right)^{2}} \quad, \quad R_{2}=\frac{R_{c}^{+}}{\left(R_{\xi}^{+}\right)^{2}}
$$

We compare our final estimates and those of [6] in table 11. We immediately observe a good overall agreement. This agreement is highly non trivial since, as discussed above, in our estimates we had to face large non universal corrections, while in the predictions of [6] only the lowest states of the spectrum were taken into account and some small discrepancies with the exact results were expected. If we trust in the overall agreement that we have found we immediately see that the major discrepancies between the two sets of data 
are in the two ratios $R_{\chi}$ and $R_{1}$, which could both be consequences of a biased estimate of $\Gamma_{-}$. It would be important to understand the reason of this discrepancy. In this respect it is worthwhile to notice that $\chi_{l}$ is the only observable for which the non-universal correction has the opposite sign with respect to the universal additive one.

\begin{tabular}{|c|c|c|}
\hline Ratio & this work & ref. [6] \\
\hline$R_{\chi}$ & $3.14(70)$ & 4.013 \\
$R_{\xi}$ & $2.19(26)$ & 1.935 \\
$R_{1}$ & $0.68(13)$ & 0.4539 \\
$R_{2}$ & $0.44(13)$ & 0.4845 \\
$R_{c}^{+}$ & $0.021(5)$ & 0.0204 \\
$R_{c}^{-}$ & $0.0068(9)$ & 0.0051 \\
$R_{\xi}^{+}$ & $0.220(20)$ & 0.2052 \\
$R_{\xi}^{-}$ & $0.100(10)$ & 0.1060 \\
\hline
\end{tabular}

Table 11: Comparison between our estimates for the universal amplitude ratios and those of ref. [6].

\section{Concluding remarks}

The aim of this paper was to test the recent predictions of [6] for various amplitude ratios in the 4 state Potts model, with the results of a high precision Monte Carlo simulation.

We made four tests on the results of our simulations:

- Comparison with exact results for small lattices.

- Comparison with the results of [21, 4] at the critical point.

- Comparison with low temperature series.

- Agreement with the duality relations.

All these tests were successfully passed. 
In looking at the scaling behaviour of our observables, we had to face a major problem, due to the presence of a marginal field in the spectrum. In performing the analysis we used the recent results of Salas and Sokal on the universal additive $\log \log / \log$ correction terms, and found in our fits the same behaviour and the same features that they reported in [4] where they looked at the finite size corrections at the critical point.

We found a relatively good overall agreement with the predictions of Cardy and Delfino with the exception of two ratios involving the low temperature susceptibility. It remains an open problem to find a more efficient way of dealing, in the analysis of Monte Carlo data, with corrections originated by the presence of marginal operators. These contributions are the probable main cause of these discrepancies.

\section{Acknowledgements}

We thank J.L.Cardy, G.Delfino, P.Dorey, F.Gliozzi and B.Nienhuis for helpful discussions. This work was partially supported by the European Commission TMR programme ERBFMRX-CT96-0045.

\section{References}

[1] M. Nauenberg and D.J. Scalapino, 'Singularities and scaling functions at the Potts model multicritical point', Phys. Rev. Lett. 44, 837 (1980).

[2] J.L. Cardy, M. Nauenberg and D.J. Scalapino, 'Scaling theory of the Potts-model multicritical point', Phys. Rev. B 22, 2560 (1980).

[3] M. Caselle and M. Hasenbusch, 'Universal amplitude ratios in the 3-D Ising model', hep-lat/9701007, J. Phys. A30 (1997) 4963.

[4] J. Salas and A.D. Sokal, 'Logarithmic corrections and finite size scaling in the two-dimensional four state Potts model', hep-lat/9607030, J.Stat.Phys 88 (1997) 567.

[5] For a comprehensive review on amplitude ratios, see for instance: A. Aharony, P.C. Hohenberg and V. Privman, 'Universal critical point amplitude relations' in 'Phase transitions and Critical phenomena' vol.14, C. Domb and J.L. Lebowitz eds. (Academic Press 1991) 
[6] J.L. Cardy and G. Delfino, 'Universal amplitude ratios in the twodimensional q-state Potts model and percolation from quantum field theory', hep-th/9712111, Nucl. Phys. B519 (1998) 551.

[7] F.A. Smirnov, 'Form factors in completely integrable models of quantum field theory', World Scientific 1992.

[8] C.M.Fortuin and P.W.Kasteleyn, 'On the random-cluster model', Physica $57,(1972) 36$.

[9] V.S. Dotsenko and V.A. Fateev, 'Conformal algebra and multipoint correlation functions in two-dimensional statistical models', Nucl. Phys. B240 (1984) 312.

[10] B. Nienhuis, 'Critical behavior of two-dimensional spin models and charge asymmetry in the Coulomb Gas', J.Stat.Phys 34 (1984) 731.

[11] A.B. Zamolodchikov, 'Integrable Field Theory from Conformal Field Theory', Proceedings of Taniguchi Symposium, Kyoto (1988)

[12] F.A. Smirnov, 'Exact S matrices for Phi(1,2) perturbated minimal models of conformal field theory', Int. J. Mod. Phys. A6 (1991) 1407.

[13] F.A. Smirnov, 'Reductions of the sine-Gordon model as a perturbation of minimal models of conformal field theory', Nucl. Phys. B337 (1990) 156.

[14] D. Bernard and A.Leclair, 'Residual Quantum symmetries of the restricted sine-Gordon theories', Nucl. Phys. B340 (1990) 721.

[15] A.B. Zamolodchikov, 'Thermodynamic Bethe ansatz for RSOS scattering theories', Nucl. Phys. B358 (1991) 497.

[16] A.B. Zamolodchikov, 'From tricritical Ising to critical Ising by thermodynamic Bethe ansatz', Nucl. Phys. B358 (1991) 524.

[17] A.B. Zamolodchikov, 'TBA equations for integrable perturbed $S U(2)_{K} \times S U(2)_{L} / S U(2)_{K+L}$ coset models', Nucl. Phys. B366 (1991) 122 . 
[18] L.Chim and A.B. Zamolodchikov, 'Integrable field theory of q-state Potts model with $0<q<4$ ', Int. J. Mod. Phys. A7 (1992) 5317.

[19] M. Karowski and P. Weisz, 'Exact form-factors in (1+1)-dimensional field theoretic models with soliton behavior', Nucl. Phys. B139 (1978) 445 .

[20] A. L. Talapov and H.W.J. Blöte, 'The magnetization of the 3-D Ising model', J. Phys. A29 (1996) 5727.

[21] J. Salas and A.D. Sokal, 'Dynamic critical behavior of a SwendsenWang type algorithm for the Ashkin-Teller model', hep-lat/9511022, J.Stat.Phys 85 (1996) 297.

[22] J.P. Straley and M.E. Fisher, 'Three-state Potts model and anomalous tricritical points', J. Phys. A6 (1973) 1310.

[23] K.M. Briggs, I.G. Enting and A.J. Guttmann, 'Series studies of the Potts model. 2. Bulk series for the square lattice', hep-lat/9312082, J. Phys. A27 (1994) 1503

[24] A.J. Liu and M.E. Fisher, 'The three-dimensional Ising model revisited numerically', Physica A 156 (1989) 35. 